\title{
Mineração
}

\section{Aperfeiçoamento da estratégia de homogeneização em pilhas chevron utilizando simulação geoestatística}

\section{(Improving blending strategies in chevron piles using geostatistical simulation)}

\author{
João Felipe Coimbra Leite Costa \\ Engenheiro de Minas, Professor, Dr., DEMIN, \\ UFRGS.E-mail: jfelipe@ufrgs.br \\ Diego Machado Marques \\ Engenheiro de Minas, UFRGS \\ E-mail:diegommarques@yahoo.com.br \\ Evandro Lino Batiston \\ Engenheiro de Minas, Vale \\ E-mail:evandro.batiston@vale.com
}

\author{
Gustavo G. Pilger \\ Engenheiro de Minas, Golder Associates \\ E-mail:gpilger@golder.com \\ Diniz Tamantini Ribeiro \\ Geólogo, Dr., Vale \\ E-mail:diniz.ribeiro@vale.com
}

Jair Carlos Koppe

Engenheiro de Minas/Geólogo, Professor, Dr., DEMIN, UFRGS.E-mail: jkoppe@ufrgs.br

\section{Resumo}

Um dos grandes problemas existentes na indústria mineira são as flutuações no teor do minério, que afetam, significativamente, o desempenho das plantas de beneficiamento. Assim, faz-se necessário um controle dessa variabilidade. Para tanto, pode-se usar combinações de minério provenientes de diferentes frentes de lavra e/ou pilhas de homogeneização. As pilhas de homogeneização são caracterizadas pela sua forma, tamanho, arranjo e número de camadas. O estudo em questão se propõe a minimizar a variabilidade dos depósitos minerais, utilizando pilhas longitudinais do tipo chevron, montadas com informações de teores do modelo de blocos gerados a partir de simulação geoestatística. As análises são feitas simulando diferentes modelos de pilhas, a partir de combinações de massas e diferentes números de camadas. Os resultados demonstram uma redução de até $90 \%$ da variabilidade, possibilitando um dimensionamento mais adequado do pátio e dos equipamentos de construção da pilha.

Palavras-chave: Homogeneização, pilhas, Simulação Seqüencial Gaussiana (SSG).

\begin{abstract}
Blending and homogenization piles are usually employed for reducing grade fluctuation from the ore feeding a processing plant. These piles are characterized by their form, size (length and height), construction lay-out and number of layers. Various methods are found for blending piles design and most fail to incorporate the in situ grade variability intrinsic of a mineral deposit. The methodology suggested in this study is able to quantify the variability of the homogenization system using multiple equally probable realizations derived from a geostatistical simulation for the grade block model. The variable tested was silica $\left(\mathrm{SiO}_{2}\right)$ in a grannulometry below $0,15 \mathrm{~mm}$ (SI3), an erratic contaminant in iron deposits. The results demonstrated a reduction exceeding $90 \%$ in the original variability (in situ compared to grades feeding the processing plant) of the system in favorable conditions. Finally, the reduction in the uncertainty in the head grades feeding the processing plant, leads to cost savings and lower economical risks.
\end{abstract}

Keywords: Homogenization, Piles, Sequential Gaussian Simulation (SGS). 


\section{Introdução}

A variabilidade dos teores de minérios que alimentam as plantas de beneficiamento afeta a otimização dos processos. Sua redução pode ser obtida pela modificação do seqüenciamento de lavra ou utilizando-se unidades de homogeneização de minério.

Nesse contexto, as pilhas de homogeneização representam o método mais simplificado e difundido utilizado para redução da variabilidade dos teores. $\mathrm{O}$ método consiste na disposição em camadas horizontais, após a britagem do minério ROM (run off mine) e da retomada posterior em fatias verticais. Desta forma, para se obterem ganhos significativos, é imprescindível a otimização dos parâmetros das pilhas, à medida que $\mathrm{o}$ incorreto dimensionamento pode acarretar em estoques elevados e, conseqüentemente, perdas financeiras. $\mathrm{O}$ incorreto dimensionamento desses parâmetros, também, pode ocasionar variabilidade de retomada da pilha em valores superiores ao esperado.

A aplicação de técnicas geoestatísticas ajuda na construção de modelos que utilizam variáveis selecionadas, interpoladas por meio de krigagem ou qualquer outra técnica de estimativa. Mas esse procedimento não pode incorporar a incerteza associada ao valor interpolado, sendo a variância dos valores estimados menor que a variância dos dados originais. Há, também, limitações associadas ao uso da variância de krigagem como uma medida da incerteza, sendo esse tópico discutido extensivamente na literatura (David, 1988; Isaaks and Srivastava, 1989). Sendo assim, a simulação geoestatística condicionada (Journel, 1974) foi utilizada nesse trabalho, pois, ao contrário da krigagem, visa a reproduzir a variabilidade in situ e a continuidade espacial do conjunto de dados.

Modelos usados deste modo reproduzem a estrutura espacial e o conjunto de dados como um todo, em lugar de prover estimativas locais seguras de um atributo em locais particulares. O mode- lo simulado é "condicionalmente simulado", se reproduzir as mesmas características de dispersão do conjunto de dados original, tal como a média, variância, covariância e o variograma. Em um modelo condicionalmente simulado, é possível endereçar perguntas que recorrem à dispersão dos graus durante a mineração ou processamento, desde que as características de dispersão dos dados originais sejam mantidas. Quanto melhor a continuidade espacial e a variabilidade do depósito puderem ser descritas, melhor numericamente será o modelo.

Nesse estudo, propõe-se a criação de uma metodologia que visa a quantificar a variabilidade dos fatores envolvidos na homogeneização de pilhas do tipo chevron, buscando um ponto ótimo de operação. Para tal, estudaram-se fatores como a variabilidade entre as pilhas (entrepilhas), na própria pilha (intrapilha) e a composição da variância do sistema.

Os dados desse estudo são provenientes das minas de Sapecado e Galinheiro, pertencentes à Vale, e se referem a um plano anual de lavra de minérios de ferro de baixo teor, itabiritos.

\section{Metodologia}

Para analisar as variáveis do sistema, utilizou-se como parâmetro a sílica $\left(\mathrm{SiO}_{2}\right)$ com granulometria inferior a 0,15 $\mathrm{mm}$, por se tratar de um contaminante bastante errático nos depósitos de ferro. Determinaram-se os teores de blocos, utilizando Simulação Seqüencial Gaussiana (SSG) (Isaaks, 1990), para se obterem múltiplos modelos equiprováveis.
Esses modelos de blocos foram submetidos ao seqüenciamento da lavra proposto pela equipe de engenharia com base no algoritmo de otimização de Lersch-Grossmann (Lerchs and Grossmann, 1965).

Dessa forma, a metodologia proposta consta de:

i. Construção de um modelo de blocos válido através de SSG.

ii. Determinação da seqüência de lavra. iii. Medida da variabilidade entrepilhas. iv. Medida da variabilidade intrapilha.

v. Composição da variabilidade do sistema.

A construção do modelo geológico e a simulação seqüencial Gaussiana dos teores dos blocos de lavra foram realizadas com auxilio do software Isatis ${ }^{\circledR}$. Para a determinação das variabilidades entrepilhas e intrapilha empregou-se um algoritmo desenvolvido em Visual Basic adaptado ao Excel ${ }^{\circledR}$ (Bolzan et. al., 2006).

O modelo de pilhas do tipo chevron é formado pela deposição de material em leitos longitudinais sobrepostos em camadas (Figura 1).

Foram escolhidos três tamanhos de pilhas, sendo o primeiro tamanho igual ao do atual pátio disponível na empresa (com capacidade aproximada, de $140.000 t$ ou aproximadamente 56 blocos de lavra). O segundo tamanho foi determinado considerando a produção de sete dias de lavra (90 blocos de lavra ou, aproximadamente, 225.000 t). O terceiro tamanho sugerido é equivalente à produção



Figura 1 - Pilha do tipo Chevron (Schofield, 1980). 
João Felipe Coimbra Leite Costa et al.

de três dias (40 blocos de lavra ou 100.000 t). Todas as pilhas foram construídas com 36 metros de largura e 14 metros de altura, variando, portanto, apenas o seu comprimento.

Considerou-se um período de um ano, no qual será lavrado um determinado número de blocos (nesse estudo 4895). Os teores de cada bloco serão diferentes entre si e diferentes da média global calculada do ano. Entretanto, quando ocorre o agrupamento dos blocos da lavra em pilhas, o teor médio da pilha (TMP) aproxima-se do teor médio anual, em comparação aos blocos que a compõem. Esse princípio, de redução da variância de dispersão com o aumento do suporte, é bem conhecido pelos geoestatísticos. Assim, as pilhas reduzem a variação dos teores que alimentam a usina, se comparados com a variabilidade de teores que se teria caso a alimentação fosse bloco a bloco. Outras considerações que podem ser feitas quanto à variância entre pilhas são as seguintes:

i. Quanto maior a pilha, menor será o desvio-padrão (variabilidade) dos teores médios das pilhas em relação ao teor médio anual. ii. Como conseqüência de (i): uma única pilha perfeitamente homogeneizada composta por todos os blocos do período teria um desvio-padrão igual à zero (em teoria).

Porém, quanto maior a pilha, maior será a dificuldade de sua operação e, conseqüentemente, os custos envolvidos serão maiores. O tamanho ideal da pilha será determinado pelo ganho em termos de redução da variabilidade que a mesma proporciona.

\section{Resultados e discussão}

Para os três tamanhos de pilhas do estudo, foram simulados 10 números de camadas, variando de um a quarenta e cinco camadas $(1,5,10,15,20,25,30,35$, 40 e 45). Como o resultado da Simulação Seqüencial Gaussiana (SSG) retornou 20 valores equiprováveis e diferentes para cada bloco de lavra, foram construídas, então, vinte diferentes pilhas de homogeneização (uma para cada modelo de blocos gerado por simulação estocástica), cada uma com até 45 camadas. Portanto, para cada pilha, de cada tamanho, foram realizadas 200 iterações (20 simulações versus 10 opções de número de camadas).

Após a validação das 20 simulações do depósito (Figura 2), foram extraídos os blocos que correspondem ao primeiro ano de lavra. Esses modelos de blocos simulados, com seus respectivos teores, foram utilizados na análise e formação de pilhas.

Utilizando o algoritmo desenvolvido, agrupam-se todos os blocos a serem lavrados num período para gerar uma simulação numérica da pilha. Depois de formada a pilha, calcula-se o teor médio da pilha. O teor médio de cada pilha é utilizado para cálculo de desvio-padrão entrepilhas, comparando o teor de cada pilha ao teor médio do ano. Testa-se o procedimento para cada tamanho de pilha, considerando todas as pilhas que podem ser formadas ao longo do ano 1 no referido tamanho. Também, a incerteza dos teores dos blocos é incorporada no processo, repetindo o processo para os 20 modelos de blocos simulados.

De posse de todos os valores de desvio-padrão para cada tamanho de pilha, pode-se fazer um gráfico de desvio-

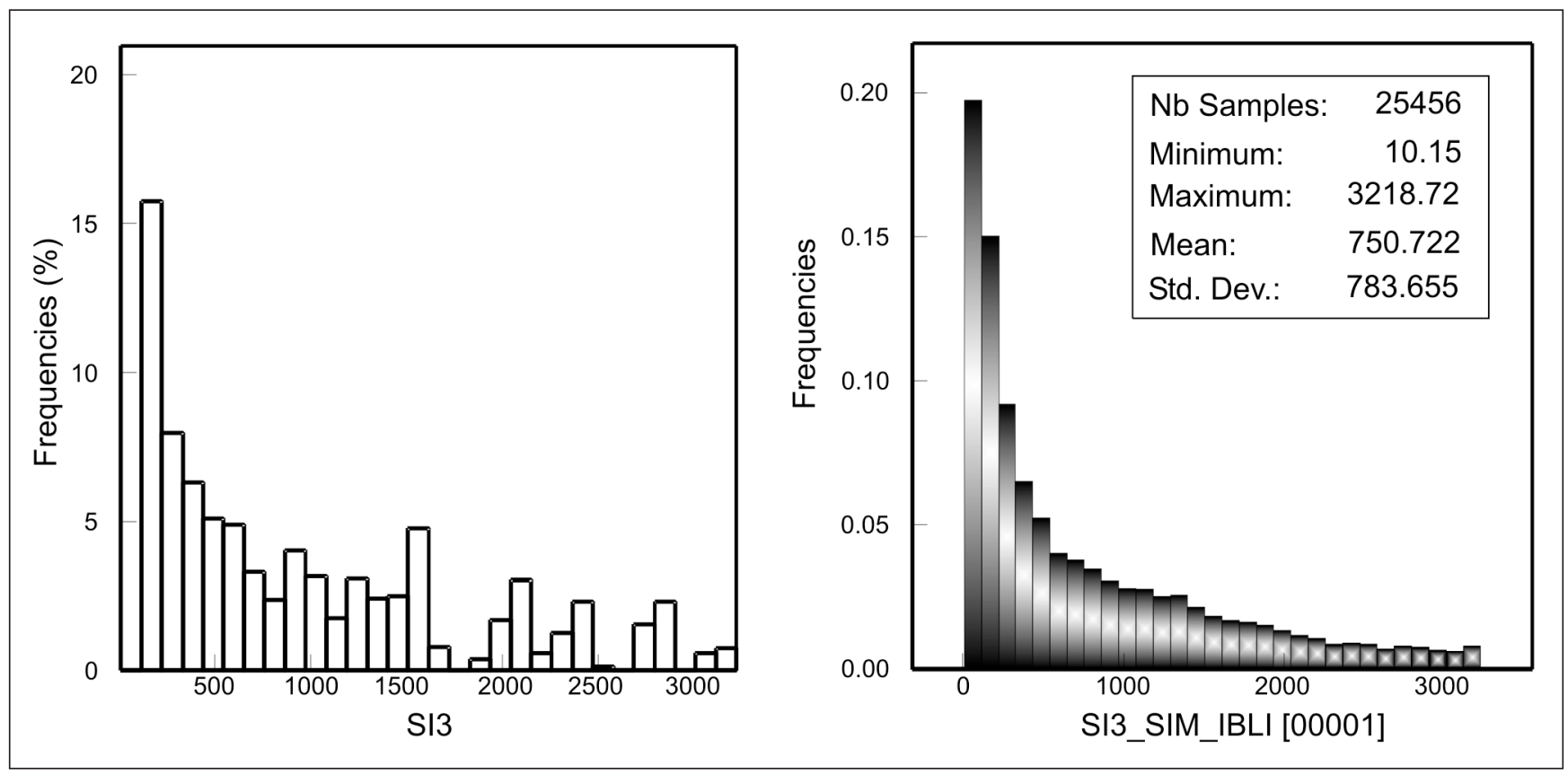

Figura 2 - (a) Histograma desagrupado dos dados originais, (b) histograma da Simulação 1. SI3 = sílica na fração inferior a 0,15 mm acumulada pela proporção retida na fração granulométrica. 
padrão versus tamanho de pilha (Figura 3 ) e obter a equação de ajuste para esta curva.

O Desvio-Padrão Teores Médio Pilhas (DPTMP), em função da massa da pilha $\left(\mathrm{m}_{\mathrm{p}}\right)$ do depósito estudado, pode ser descrito pela seguinte equação:

$\mathrm{DPTM}=12,16 \mathrm{~m}_{\mathrm{p}}^{-0,3062}$

A variabilidade intrapilha é obtida calculando o desvio-padrão do teor de cada seção vertical da pilha retomada a cada metro em relação ao teor médio da pilha. Esse desvio-padrão dos teores de retomada está associado ao número de camadas utilizado na construção da pilha. Observa-se que, para cada ano de lavra, haverá um número variável de pilhas a serem formadas em função do tamanho de pilha escolhido.

Após essa fase, o processo foi repetido para os demais cenários fornecidos pelos dados estocásticos (demais modelos de blocos equiprováveis). Esse processo, então, se repete para as 200 possíveis pilhas que podem ser formadas com um dado número de blocos de lavra (20 modelos de blocos distintos com 10 opções de número de camadas).

$\mathrm{Na}$ Figura 4, estão registrados os resultados do desvio-padrão intrapilha para as 20 simulações dos teores dos blocos para as diversas pilhas a serem formadas no ano 1, cada uma com uma massa de 225.000 t. Nota-se que há uma estabilização da variabilidade em, aproximadamente, 10 camadas. Na mesma figura, temos os valores máximos, médios e mínimos de todas as pilhas simuladas com 225.000 t e a equação do modelo de ajuste exponencial para a curva média.

O Desvio-Padrão Médio Intrapilhas (DPMIP), para pilhas de $225.000 \mathrm{t}$, em função do número de camadas $(\mathrm{N})$ do depósito estudado, pode ser descrito pela seguinte equação:

DPMIP $=16,25 N^{-0,6891}$

Portanto podemos constatar que a variabilidade intrapilha está associada a:

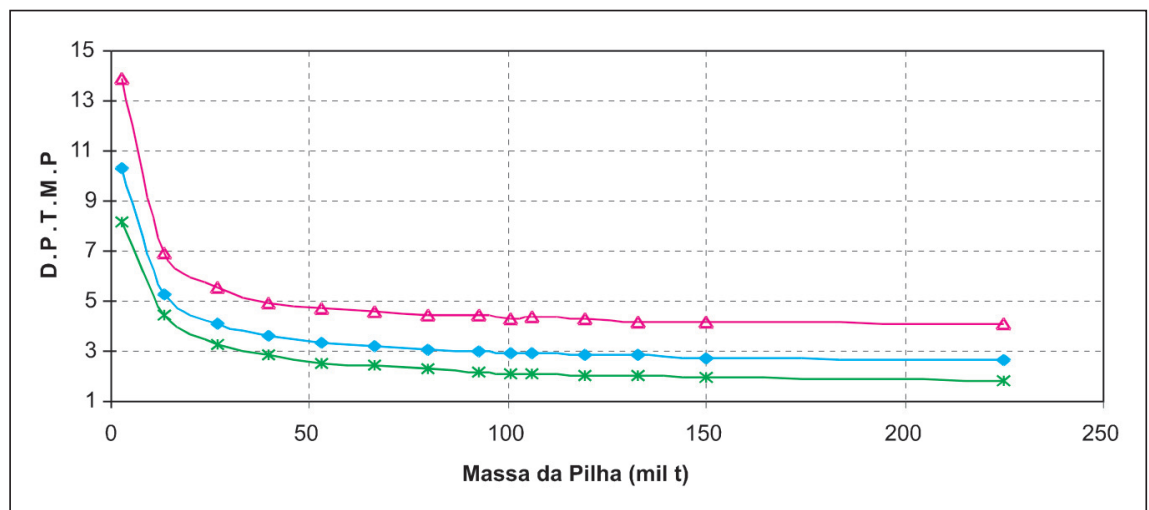

Figura 3 - Desvio-padrão entrepilhas: máximo, médio e mínimo, considerando os 20 modelos de blocos obtidos por simulação geoestatística.

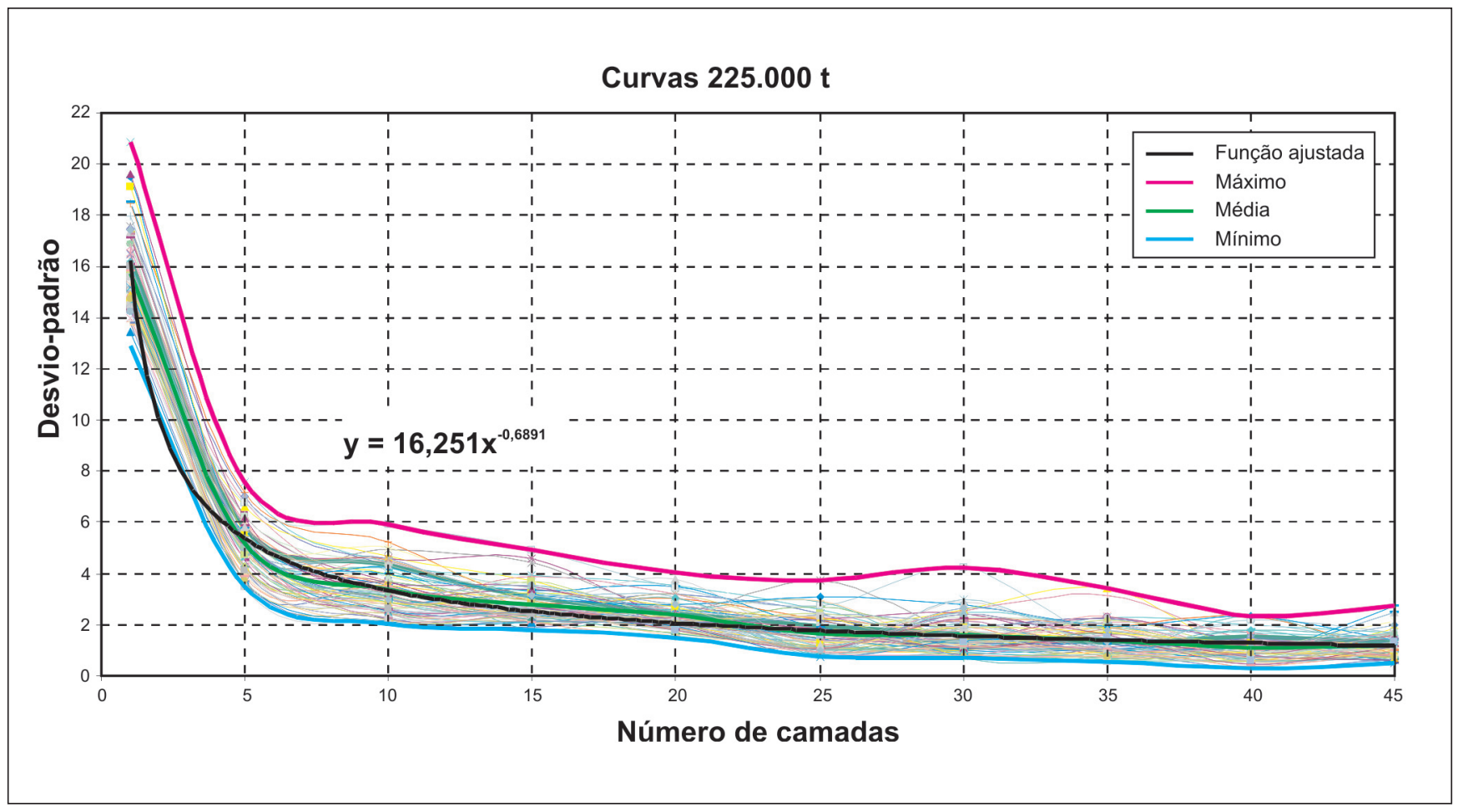

Figura 4 - Desvio-padrão das simulações, máximo, médio e mínimo para 20 simulações em função do número de camadas em pilhas de $225.000 \mathrm{t}$, considerando todas as possíveis pilhas do ano 1 . 
- Variabilidade dos teores dos blocos que compõem a pilha.

- Variabilidade interna do bloco.

- Seqüência de lavra.

- Massa da pilha.

- Número de camadas.

Sendo assim, a variabilidade do sistema de homogeneização é a soma de todos os desvios-padrão presentes no sistema, podendo ser considerada como a soma de todas as incertezas associadas ao processo. Após a quantificação destas, pode-se montar uma tabela que associa todas essas variâncias em função do número de camadas e da massa da pilha. (Tabela 1).

A Tabela 2 mostra os resultados médios obtidos para o estudo em questão.

\section{Conclusões}

Por meio da metodologia adotada, constatou-se que é possível reduzir, em até $90 \%$, a variabilidade dos teores que irão alimentar a unidade de beneficiamento de minério, em comparação à variabilidade inicial do sistema.

Recomenda-se realizar o processo de simulação a partir de blocos pequenos (mais próximo do suporte de alimentação da pilha). Entretanto, para isso, é necessário adensar a malha de amostragem, visando a uma melhor qualidade no condicionamento das simulações.

Mesmo com a homogeneização perfeita, ainda haverá variação na retomada, devido às variações dos teores ao longo do bloco. Quanto mais homogêneo e menor for o bloco, mais constantes serão os teores dos elementos discretos que o compõem e, portanto, mais constantes serão os teores de retomada.

A operação de um sistema de homogeneização traz diferentes ganhos na redução da variabilidade, dependendo do modo como é utilizado. O usuário deve escolher os parâmetros da pilha (tamanho e número de camadas) que satisfaçam as exigências de variabilidade máximas admitidas requeridas nas próximas etapas do processo.

A possibilidade de uma dupla fase de homogeneização, em pilhas pequenas e de maior número de camadas, deveria ser considera como fator a ser avaliado em projetos futuros.

Tabela 1 - Composição da variabilidade do sistema de homogeneização.

\begin{tabular}{|c|c|c|c|c|c|}
\hline \multirow{2}{*}{$\begin{array}{c}\text { Massa da } \\
\text { Pilha }\end{array}$} & \multirow{2}{*}{ Desvio-padrão } & \multicolumn{4}{|c|}{ Número de Camadas } \\
\hline & & N1 & N2 & $\ldots$ & $\mathrm{Nn}$ \\
\hline \multirow{3}{*}{$M_{1}$} & Entrepilhas & DPEP $_{M 1}$ & DPEP $_{M 1}$ & & DPEP $_{M 1}$ \\
\hline & Intrapilha & $\operatorname{DPIP}_{\mathrm{N} 1}$ & DPIP $_{\mathrm{N} 2}$ & & DPIP $_{\mathrm{Nn}}$ \\
\hline & Sistema & $\mathrm{DPEP}_{\mathrm{M} 1}+\mathrm{DPIP}_{\mathrm{N} 1}$ & $\mathrm{DPEP}_{\mathrm{M} 1}+\mathrm{DPIP}_{\mathrm{N} 2}$ & & $\mathrm{DPEP}_{\mathrm{M} 1}+\mathrm{DPIP}_{\mathrm{Nn}}$ \\
\hline \multirow{3}{*}{$\mathbf{M}_{2}$} & Entrepilhas & DPEP $_{M 2}$ & DPEP $_{M 2}$ & & DPEP $_{M 2}$ \\
\hline & Intrapilha & $\mathrm{DPIP}_{\mathrm{N} 1}$ & $\mathrm{DPIP}_{\mathrm{N} 2}$ & & $\operatorname{DPIP}_{\mathrm{Nn}}$ \\
\hline & Sistema & $\mathrm{DPEP}_{\mathrm{M} 2}+\mathrm{DPIP}_{\mathrm{N} 1}$ & $\mathrm{DPEP}_{\mathrm{M} 2}+\mathrm{DPIP}_{\mathrm{N} 2}$ & & $\mathrm{DPEP}_{\mathrm{M} 2}+\mathrm{DPIP}_{\mathrm{Nn}}$ \\
\hline \multicolumn{6}{|l|}{$\vdots$} \\
\hline \multirow{3}{*}{$M_{n}$} & Entrepilhas & DPEP $_{M n}$ & DPEP $_{M n}$ & & DPEP $_{M n}$ \\
\hline & Intrapilha & $\operatorname{DPIP}_{\mathrm{N} 1}$ & $\operatorname{DPIP}_{\mathrm{N} 2}$ & & $\operatorname{DPIP}_{N n}$ \\
\hline & Sistema & $\mathrm{DPEP}_{\mathrm{Mn}}+\mathrm{DPIP}_{\mathrm{N} 1}$ & $\mathrm{DPEP}_{\mathrm{Mn}}+\mathrm{DPIP}_{\mathrm{N} 2}$ & & $\mathrm{DPEP}_{\mathrm{Mn}}+\mathrm{DPIP}_{\mathrm{Nn}}$ \\
\hline
\end{tabular}

onde: DPEP = Desvio-Padrão Entrepilhas (máximo, médio ou mínimo).

DPIP = Desvio-Padrão Intrapilha (máximo, médio ou mínimo).

$\mathrm{M}_{\mathrm{n}}=$ Massa da pilha.

$\mathrm{N}_{\mathrm{n}}=$ Número de camadas utilizadas. 
Tabela 2 - Resultados médios do sistema de homogeneização.

\begin{tabular}{|c|c|c|c|c|c|c|c|c|c|c|c|}
\hline \multirow{2}{*}{$\begin{array}{l}\text { Massa da } \\
\text { Pilha }\end{array}$} & \multirow{2}{*}{ Desvio-Padrão } & \multicolumn{10}{|c|}{ Número de camadas } \\
\hline & & 1 & 5 & 10 & 15 & 20 & 25 & 30 & 35 & 40 & 45 \\
\hline \multirow{3}{*}{100.000} & Entrepilhas & 2,9 & 2,9 & 2,9 & 2,9 & 2,9 & 2,9 & 2,9 & 2,9 & 2,9 & 2,9 \\
\hline & Intrapilha & 14,5 & 4,7 & 2,9 & 2,2 & 1,8 & 1,5 & 1,3 & 1,2 & 1,1 & 1,0 \\
\hline & Sistema & 17,4 & 7,6 & 5,8 & 5,1 & 4,7 & 4,4 & 4,2 & 4,1 & 4,0 & 3,9 \\
\hline \multirow{3}{*}{150.000} & Entrepilhas & 2,7 & 2,7 & 2,7 & 2,7 & 2,7 & 2,7 & 2,7 & 2,7 & 2,7 & 2,7 \\
\hline & Intrapilha & 14,5 & 4,7 & 2,9 & 2,2 & 1,8 & 1,5 & 1,3 & 1,2 & 1,1 & 1,0 \\
\hline & Sistema & 17,2 & 7,4 & 5,6 & 4,9 & 4,5 & 4,2 & 4,0 & 3,9 & 3,8 & 3,7 \\
\hline \multirow{3}{*}{225.000} & Entrepilhas & 2,6 & 2,6 & 2,6 & 2,6 & 2,6 & 2,6 & 2,6 & 2,6 & 2,6 & 2,6 \\
\hline & Intrapilha & 14,5 & 4,7 & 2,9 & 2,2 & 1,8 & 1,5 & 1,3 & 1,2 & 1,1 & 1,0 \\
\hline & Sistema & 17,1 & 7,3 & 5,5 & 4,8 & 4,4 & 4,1 & 3,9 & 3,8 & 3,7 & 3,6 \\
\hline
\end{tabular}

\section{Referências bibliográficas}

BOLZAN, I.P., COSTA, J.F.C.L., RIBEIRO, D.T., PILGER, G.G., BATISTON, E.L., MARQUES, D.M., KOPPE, J. Aperfeiçoamento da estratégia de homogeneização de minérios utilizando simulação geoestatística. Laboratório de pesquisa mineral e planejamento mineiro, UFRGS, 2006. 51p. (Relatório interno).
DAVID, M. Handbook of applied advanced geostatistical ore reserve estimation. Amsterdam: Elsevier Science Publisher, 1988. 216p.

ISAAKS, E.H. The application of Monte Carlo methods to the analysis of spatially correlated data. USA: Stanford University, 1990. 213p. (PhD. Thesis)

ISAAKS, E.H., SRIVASTAVA, M.R. An introduction to applied geostatistics. New York: Oxford University Press, 1989. 561p.

JOURNEL, A. G. Geostatistics for conditional simulation of ore bodies. Economic Geology, v. 69, n. 5, p. 673-687, 1974.

LERCHS, H., GROSSMANN, I. F. Optimum design of open-pit mines, CIM Bulletin, v. 58 , p. $47-54,1965$.

SCHOFIELD, C.G. Homogenization/blending systems design and control for minerals processing. Germany: TransTech Publications, 1980. 236p.

Artigo recebido em 12/09/2007 e aprovado em 01/03/2008. 